\title{
Disappearance of giant cells and presence of newly formed bone in the pulmonary metastasis of a sacral giant-cell tumor following denosumab treatment: A case report
}

\author{
TETSURO YAMAGISHI ${ }^{1}$, HIROYUKI KAWASHIMA ${ }^{1}$, AKIRA OGOSE $^{1}$, TARO SASAKI ${ }^{1}$, \\ TETSUO HOTTA $^{1}$, SHOICHI INAGAWA ${ }^{2}$, HAJIME UMEZU ${ }^{3}$ and NAOTO ENDO ${ }^{1}$ \\ ${ }^{1}$ Division of Orthopedic Surgery, Graduate School of Medical and Dental Sciences, Niigata University; \\ Divisions of ${ }^{2}$ Radiology and ${ }^{3}$ Pathology, Niigata University Medical and Dental Hospital, Niigata 951-8510, Japan
}

Received November 11, 2014; Accepted September 9, 2015

DOI: $10.3892 / \mathrm{ol} .2015 .3858$

\begin{abstract}
A giant-cell tumor of the bone (GCTB) is a benign but locally aggressive bone tumor. Recently, the receptor activator of nuclear factor $\kappa \mathrm{B}$ (RANK) ligand inhibitor, denosumab, has demonstrated activity against giant-cell tumors. The current study reports a case of a sacral GCTB with lung metastasis. A 19-year-old male patient presented with right buttock pain and right lower leg pain, and a sacral GCTB was diagnosed based on the histological analysis of a biopsy specimen. The patient was successfully treated with neoadjuvant denosumab therapy, which allowed curettage. In addition, the pulmonary nodule reduced in size following denosumab administration, and surgical resection was performed. Since the operation, the patient has been managed with the continued use of denosumab with no sign of recurrence. Microscopic findings from the surgical specimen following denosumab treatment revealed that the giant cells had disappeared and woven bone had formed. The specimen from the pulmonary nodule exhibited similar findings to the surgical specimen. It was reported that denosumab treatment was able to reduce the number of giant cells and RANK-positive stromal cells, and cause the formation of new bone in the primary lesion. The present study reports the first case to demonstrate the efficiency of denosumab in treating pulmonary metastasis of GCTB.
\end{abstract}

\section{Introduction}

A giant-cell tumor of the bone (GCTB) is a benign but aggressive skeletal neoplasm, with a high recurrence rate $(1,2)$ of between 33 and $50 \%(3,4)$. Therefore, it is desirable to perform

Correspondence to: Dr Hiroyuki Kawashima, Division of Orthopedic Surgery, Graduate School of Medical and Dental Sciences, Niigata University, Asahimachi 1-751, Niigata 951-8510, Japan E-mail: inskawa@med.niigata-u.ac.jp

Key words: bone formation, giant-cell tumor of the bone, denosumab, receptor activator of nuclear factor $\kappa \mathrm{B}$ ligand, metastasis en bloc resection as a radical cure. However, surgical interventions, including curettage and resection, are associated with significant complications and recurrence rates. The treatment of GCTB is controversial $(3,5)$. Partial and/or total sacrectomy is the ideal curative treatment. However, GCTB in the spine, pelvis and sacrum present as massive tumors and the size and location make surgical treatment difficult and dangerous (5). It can result in significant morbidity, including neurological complication and infection, and mortality (6). In addition, GCTBs have abundant vessels that often cause massive bleeding during surgery (3). Therefore, it is difficult to treat GCTBs of the sacrum. The survival rate to recurrence is $90 \%$ at 60 and 120 months (7). Survival to local recurrence with and without radiation was $91 \%$ and $86 \%$, with and without embolization was $91 \%$ and $86 \%$, and with and without local adjuvants $88 \%$ and $92 \%$, respectively (7). However, a number of treatment modalities have been reported. The standard treatment is aggressive curettage or wide resection. If complete resection cannot be easily achieved, preoperative arterial embolization, intralesional curettage, or radiation therapy are alternatives (7). However, the best treatment for GCTB of the sacrum remains controversial and unclear. Lung metastasis from a GCTB is rare; it is reportedly observed in $2-10 \%$ of patients with GCTB $(8,9)$.

Denosumab is a fully human monoclonal antibody against the receptor activator of nuclear factor $\kappa \mathrm{B}$ (RANK) ligand (RANKL). RANKL facilitates the differentiation of osteoclast precursors into osteoclasts, resulting in bone resorption (10). This is important in the regulation of bone growth and turnover. In a phase II study of adults with giant-cell tumors, significant clinical response to denosumab was observed (11): The clinical response refers to the elimination of giant cells histologically in $20 / 35$ patients who were administrated denosumab. Denosumab was approved for the treatment of GCTBs in June 2014 in Japan. The effect of denosumab treatment on patients with GCTBs has previously been reported; $90 \%$ of GCTB patients derived clinical benefit from denosumab, such as functional status or pain (12).

The current study presents the case of a sacral GCTB with pulmonary metastasis, which was treated with curettage and denosumab. This case demonstrates the effect of denosumab on pulmonary metastasis from GCTB. 
A

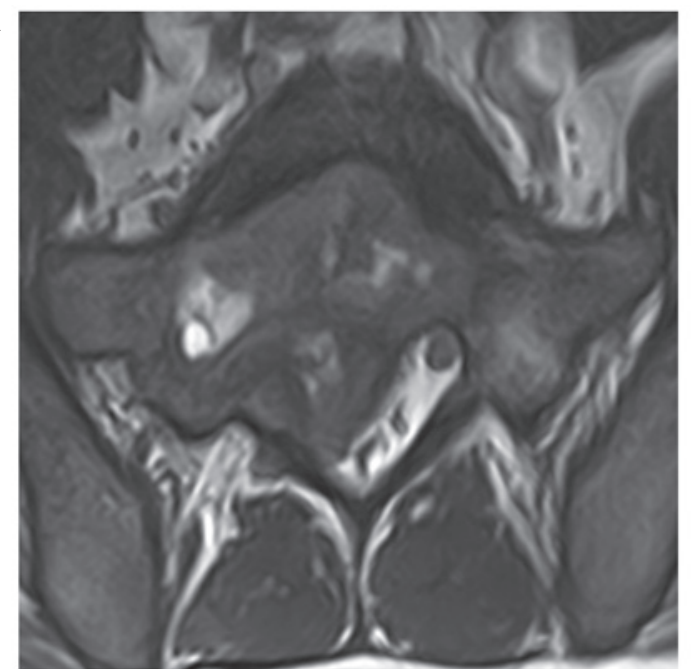

B

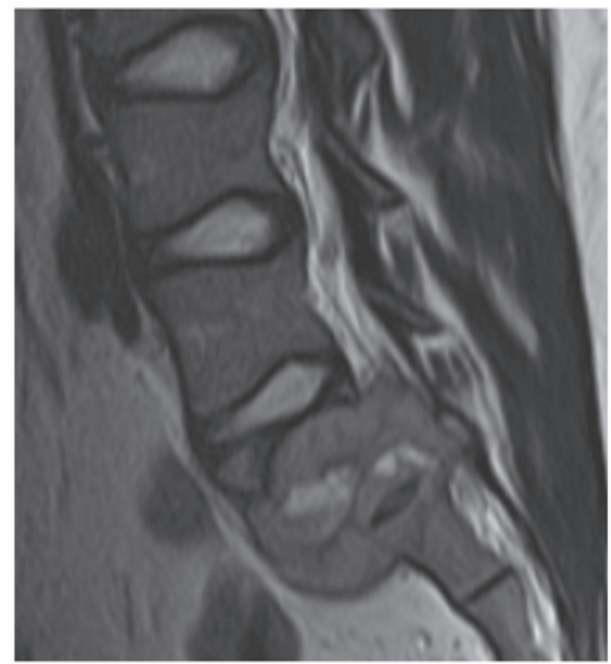

Figure 1. (A) A coronal T2-weighted magnetic resonance image showing the tumor extending to the lumbar canal. (B) A sagittal T1-weighted magnetic resonance image showing a giant-cell tumor extending from sacral vertebra S1-S2.

$\mathbf{A}$

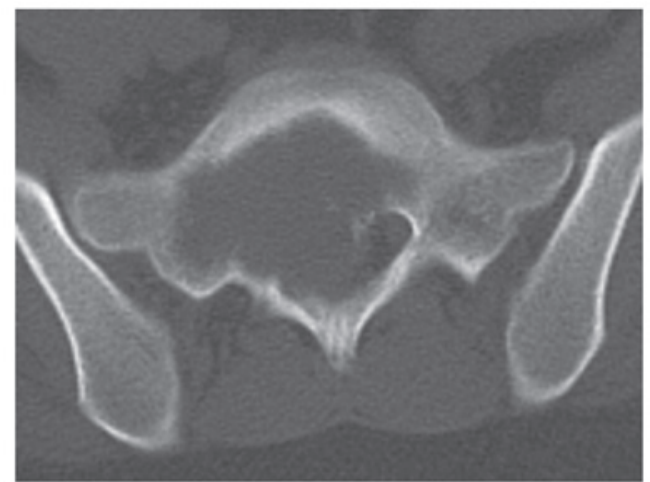

B

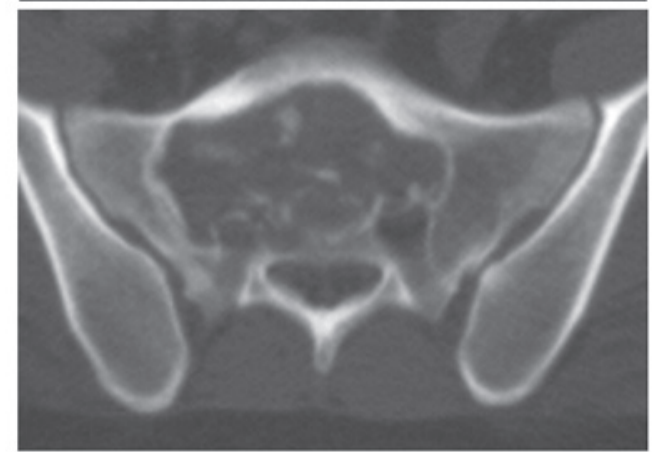

C

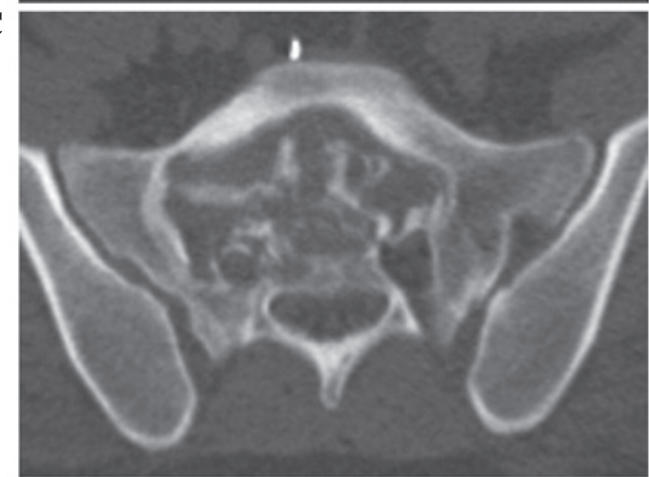

Figure 2. CT imaging of the sacrum. (A) Sacral giant-cell tumor exhibiting an osteolytic lesion. (B) After 4 months of denosumab treatment, CT imaging of the sacrum revealed osteogenesis. (C) After 8 months of denosumab treatment, CT imaging revealed progression of osteogenesis, and preoperative vascular embolization was performed. CT, computed tomography.
A

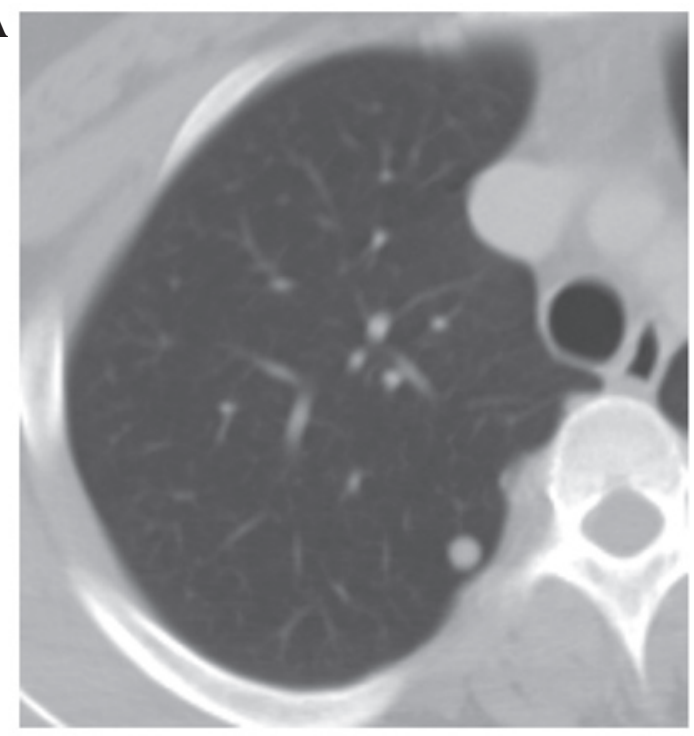

B

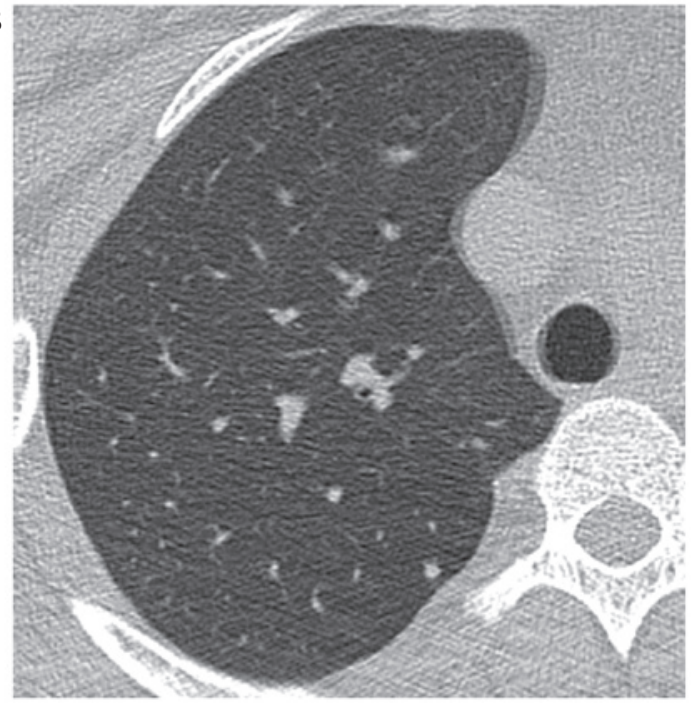

Figure 3. (A) Enhanced chest CT imaging revealed a right pulmonary nodule measuring $6 \mathrm{~mm}$ in diameter, which was confirmed to be a metastatic lesion. (B) After 10 months of denosumab treatment, CT imaging revealed that the pulmonary nodule had reduced in size. CT, computed tomography. 


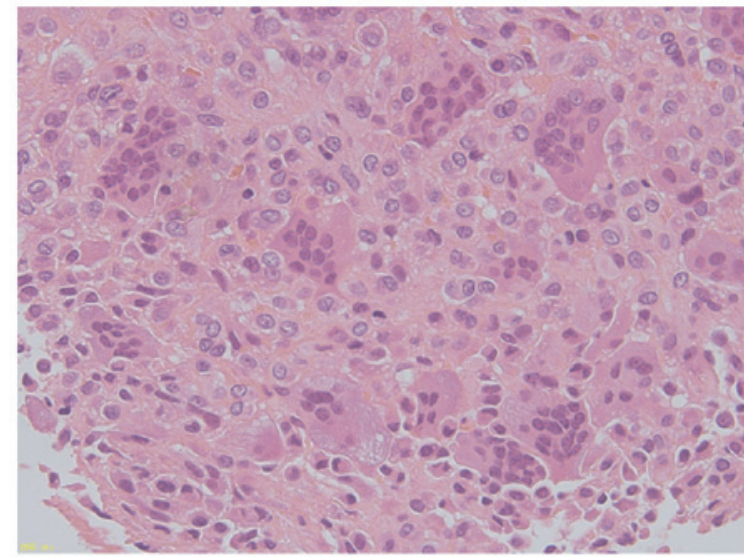

Figure 4. Photomicrograph showing a hematoxylin and eosin-stained tissue specimen of the sacral giant-cell tumor. Note the omnipresent multinucleated giant cells. Magnification, x200.

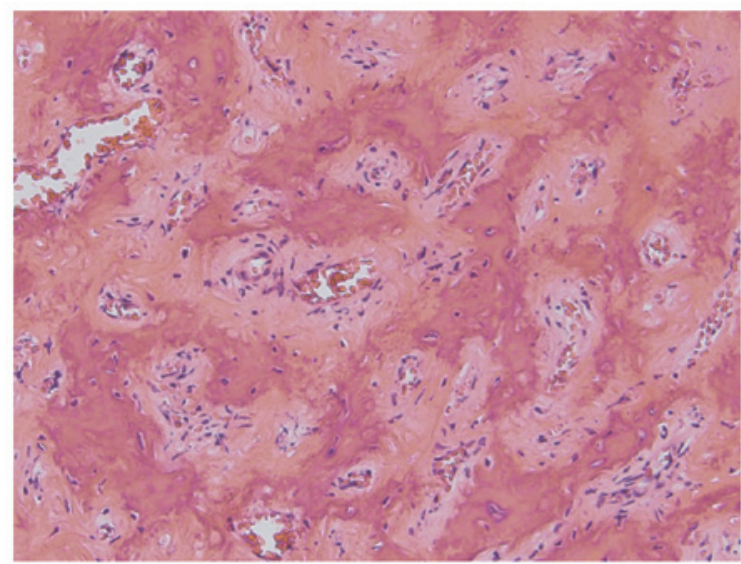

Figure 5. Photomicrograph of the sacral giant-cell tumor. Multinucleated giant cells are not present; formation of osteoid and woven bones can be observed (hematoxylin and eosin staining). Magnification, x100.

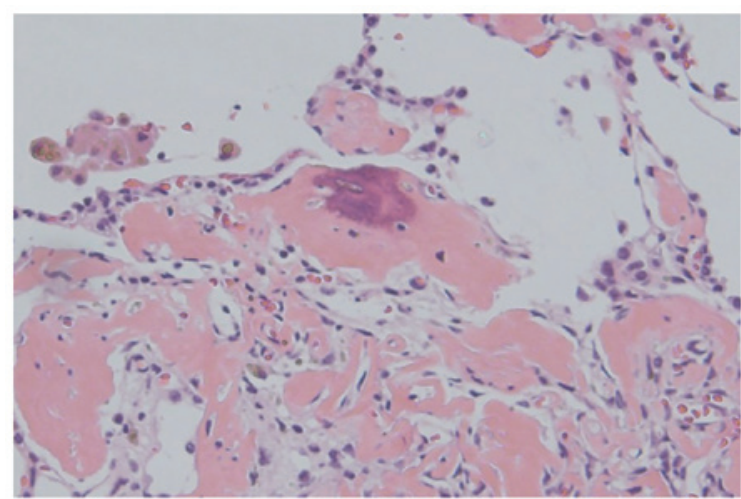

Figure 6. Photomicrograph of the pulmonary nodule showing the formation of osteoid and woven bone. Multinucleated giant and atypical stromal cells are not observed (hematoxylin and eosin staining). Magnification, x200

\section{Case report}

A 19-year-old male patient presented to Niigata University Medical and Dental Hospital (Niigata, Japan) in January 2013 complaining of right buttock pain and right lower leg pain.
Initial magnetic resonance imaging revealed a bone tumor in the sacrum (Fig. 1). Computed tomography (CT) scans of the sacrum (Fig. 2) and lungs (Fig. 3) were performed. This revealed an osteolytic lesion in the sacrum (Fig. 2A). In addition, enhanced chest $\mathrm{CT}$ revealed a right pulmonary nodule, measuring $6 \mathrm{~mm}$ in diameter, which was suspected to be metastasis from GCTB (Fig. 3A). An open biopsy of the sacrum was performed, and analysis of the specimen revealed that it consisted of stromal cells and multinucleated osteoclast giant cells, leading to a diagnosis of GCTB (Fig. 4). Initially, the treatment plan included preoperative embolization and surgical treatment. Embolization with polyvinyl alcohol was performed twice. Following embolization, the buttock pain improved.

The patient subsequently received $120 \mathrm{mg}$ of denosumab once per month, commencing in January 2013, as neoadjuvant therapy for the sacral tumor and the pulmonary nodule. Follow-up CT after 8 months of treatment, revealed gradual osteogenesis in the sacral tumor over time (Fig. 2B). In September 2013, follow-up CT revealed marked osteogenesis, and the tumor was judged to be stable; surgery was subsequently planned (Fig. 2C). Following preoperative embolization with micro-coils (Tornado ${ }^{\circledR}$; Kook Japan, Tokyo, Japan), curettage of the tumor was undertaken with laminectomy at the L5-S1 spinal segments. Histopathological examination revealed an immature trabecular bone, and osteoclast-type giant cells were not observed in the sacral specimen (Fig. 5).

Following surgery, the patient has continued to receive $120 \mathrm{mg}$ denosumab once per month. After 10 months of denosumab treatment, a chest CT scan (performed in December 2013) indicated that the pulmonary nodule had reduced in size sufficiently to allow curative surgery, which was subsequently performed in January 2014 (Fig. 3B). The nodule was resected via video-assisted thoracoscopic surgery. Histopathologically, osteoclast-type giant cells or spindle cells were not observed in the pulmonary nodule, while osteoids were observed (Fig. 6). At the follow-up examination 6 months later, CT imaging did not show any tumor progression; however, ossification was observed in the sacrum. This was considered to be an effect of the denosumab treatment. The patient is currently 20 months into the treatment, and has no buttock pain. The patient has received $120 \mathrm{mg} / \mathrm{month}$ denosumab after surgical treatment. Present CT scan revealed no evidence of recurrence. Further treatment is not planned at present, however the interval of denosumab administration may be extended according to symptoms or follow up CT scans in the future.

\section{Discussion}

Systemic treatment with denosumab as neoadjuvant therapy has been demonstrated to successfully reduce the surgical morbidity of tumor resection, and leads to osteoclast absence and reactive bone formation (13). In the present study, curettage of the GCTB was conducted following denosumab neoadjuvant therapy, with a small amount of bleeding. Denosumab exerts its effects by binding to RANKL, thus blocking its binding to RANK on osteoclasts and osteoclast precursors. This inhibits the differentiation of osteoclasts and osteoclast-mediated bone resorption. As the giant cells in GCTB express RANK, 
denosumab is a promising target-specific therapy for this tumor (14,15). Thomas et al (11) reported the result of an open-label, phase II study with single-agent denosumab in patients who were aged $>18$ years and had recurrent giant-cell tumors or unresectable tumors. Following 25 weeks of treatment, $86 \%$ of patients met tumor response criteria (11).

Branstetter et al (12) reported that denosumab treatment for patients with GCTBs significantly reduced RANK-positive giant cells and stromal cells, and promoted bone formation at the primary lesion. In the present study, compared with the open biopsy and resected sacral specimens, giant cells disappeared following denosumab treatment, and bone formation was observed. Furthermore, the chest CT scan revealed that the pulmonary nodule reduced in size following denosumab treatment; the nodule from which the pathological examination specimen was taken demonstrated bone formation, while giant cells were not observed in the pulmonary specimen. Akaike et al (16) reported local recurrence of a GCTB following treatment with denosumab, in which bone formation was observed histologically and multinucleated giant cells had disappeared. Histological changes following denosumab treatment were reported in the soft tissue of the GCTB, but not in the pulmonary metastasis from GCTB. The case descibed by Akaike et al (16) was the first case to demonstrate the efficiency of denosumab in treating a pulmonary metastasis from GCTB.

In the present study, diagnosis of the pulmonary lesion as metastasis from GCTB was difficult; the differential diagnoses of this nodule included metastasis from GCTB, osteosarcoma and undifferentiated pleomorphic sarcoma. As giant cells were not observed and stromal cells exhibited little cytological atypia in the pulmonary specimen, the bone formation in the pulmonary nodule was thought to be non-neoplastic but rather reactive bone formation and, therefore, the pulmonary nodule was diagnosed as a metastasis of the GCTB.

Certain patients may require long-term denosumab treatment; however its toxicity profile raises concerns regarding long-term use (13). The optimal duration of denosumab treatment is unknown. In addition, Skubitz et al (17) reported that recurrences following apparent complete responses have been observed after the discontinuation of denosumab treatment. Another problem is monitoring the patient for complications and recurrence after discontinuing the administration of denosumab $(14,18)$. Long-term follow-up is necessary as long-term treatment has not been attempted previously. Denosumab may be an effective option in cases in which surgical treatment is associated with high morbidity.

In conclusion, denosumab suppresses osteoclast differentiation and reduces bone resorption. In the present study, denosumab demonstrated efficacy against pulmonary metastasis of GCTB. Microscopic findings from pulmonary specimens following denosumab treatment revealed the disappearance of giant cells and the presence of newly formed bone.

\section{References}

1. Schwartz HS: Giant cell tumor of bone. Compr Ther 19: 64-68, 1993.

2. Mendenhall WM, Zlotecki RA, Scarborough MT, Gibbs CP and Mendenhall NP: Giant cell tumor of bone. Am J Clin Oncol 29: 96-99, 2006

3. Turcotte RE, Sim FH and Unni KK: Giant cell tumor of the sacrum. Clin Orthop Relat Res 291: 215-221, 1993.

4. Randall RL: Giant cell tumor of the sacrum. Neurosurg Focus 15: E13, 2003.

5. Lin PP, Guzel VB, Moura MF, Wallace S, Benjamin RS, Weber KL, Morello FA Jr, Gokaslan ZL and Yasko AW: Long-term follow-up of patients with giant cell tumor of the sacrum treated with selective arterial embolization. Cancer 95: 1317-1325, 2002

6. Doita M, Harada T, Iguchi T, Sumi M, Sha H, Yoshiya S and Kurosaka M: Total sacrectomy and reconstrction for sacral tumors. Spine 28: E296-301, 2003

7. Ruggieri P, Mavrogenis AF, Ussia G, Angelini A, Papagelopoulos PJ and Mercuri M: Recurrence after and complications associated with adjuvant treatment for sacral giant cell tumor. Clin Orthop Relat Res 468: 2954-2961, 2010.

8. Yip KM, Leung PC and Kumta SM: Giant cell tumor of bone. Clin Orthop Relat Res 323: 60-64, 1996.

9. Malawer MM, Bickels J, Meller I, Buch RG, Henshaw RM and Kollender Y: Cryosurgery in the treatment of giant cell tumor. A long-term followup study. Clin Orthop Relat Res 359: 176-188, 1999 .

10. Bekker PJ, Holloway DL, Rasmussen AS, Murphy R, Martin SW, Leese PT, Holmes GB, Dunstan CR and DePaoli AM: A single-dose placebo-controlled study of AMG 162, a fully human monoclonal antibody to RANKL, in postmenopausal women. J Bone Miner Res 19: 1059-1066, 2004.

11. Thomas D, Henshaw R, Skubitz K, Chawla S, Staddon A, Blay JY, Roudier M, Smith J, Ye Z, Sohn W, et al: Denosumab in patients with giant-cell tumour of bone: An open-label, phase 2 study. Lancet Oncol 11: 275-280, 2010.

12. Branstetter DG, Nelson SD, Manivel JC, Blay JY, Chawla S, Thomas DM, Jun S and Jacobs I: Denosumab induces tumor reduction and bone formation in patients with giant-cell tumor of bone. Clin Cancer Res 18: 4415-4424, 2012.

13. Mak IW, Evaniew N, Popovic S, Tozer R and Ghert M: A translational study of the neoplastic cells of giant cell tumor of bone following neoadjuvant denosumab. J Bone Joint Surg Am 96: Aug 6, 2014 (Epub ahead of print).

14. Lange T, Stehling C, Fröhlich B, Klingenhöfer M, Kunkel P, Schneppenheim R, Escherich G, Gosheger G, Hardes J, Jürgens H and Schulte TL: Denosumab: A potential new and innovative treatment option for aneurysmal bone cysts. Eur Spine 22:1417-1422, 2013.

15. Akeda K, Kasai Y, Sakakibara T, Matsumine A, Takegami N, Yamada J and Sudo A: Effect of denosumab on recurrent giant cell reperative granuloma of the lumber spine. Spine 40:E601-608, 2015.

16. Akaike K, Suehara Y, Takagi T, Kaneko K and Saito T: An eggshell-like mineralized recurrent lesion in the popliteal region after treatment of giant cell tumor of the bone with denosumab. Skeletal Radiol 43: 1767-1772, 2014.

17. Skubitz KM: Giant cell tumor of bone: Current treatment options. Curr Treat Options Oncol 15: 507-518, 2014.

18. Karras NA, Ogilvie C, Manivel C, Skubitz KM and Lipsitz E: Denosumab treatment of metastatic giant-cell tumor of bone in a 10-year-old girl. J clin Oncol 31: e200-e202, 2013. 